Hautarzt 2012 - 63 [Suppl 1]:5-7

DOI 10.1007/s00105-011-2290-y

c) Springer-Verlag 2012
T. Ruzicka' $\cdot$ M. Meurer ${ }^{2}$

${ }^{1}$ Klinik und Poliklinik für Dermatologie und Allergologie, Klinikum

der Ludwig-Maximilians-Universität München

${ }^{2}$ Klinik und Poliklinik für Dermatologie, Universitäts Allergie Centrum, Technische Universität Dresden

\title{
Gratulation zum 90. Geburtstag von Professor Dr. med. Dr. h.c. mult. Otto Braun-Falco
}

Am 25.04.2012 vollendet Professor Dr. med. Dr. h.c. mult. Otto Braun-Falco seinen 90. Geburtstag. Mit unserer Zeitschrift Der Hautarzt ist der Doyen der Deutschen Dermatologie mehr als 50 Jahre verbunden, seit 1961 als Mitarbeiter des damaligen Herausgebers Prof. Marchionini, von 1968 bis 1993 als Schriftleiter und Hauptherausgeber, davon viele Jahre zusammen mit Prof. Petzoldt, Heidelberg, seit 1993 als Ehrenherausgeber - Grund genug für die beiden Münchner bzw. ExMünchner im derzeitigen Herausgebergremium, der bedeutendsten Persönlichkeit der jüngsten deutschen und internationalen dermatologischen Geschichte eine Sonderbeilage von Der Hautarzt als Festschrift zu widmen! Allen Schülern und Weggefährten des Jubilars, die zu dieser Festschrift in hervorragender Weise beigetragen haben, sei im Namen des Herausgebergremiums ebenso herz lich gedankt wie Frau Dr. Wieland und Frau Dr. Kind vom Springer-Verlag für die enthusiastische Unterstützung dieser Festschrift.

Mit Dankbarkeit begehen die Deutsche Dermatologie und die Klinik für Dermatologie und Allergologie der Ludwig-Maximilians-Universität München diesen Festtag, denn sie verdanken Otto Braun-Falco unendlich viel. Man braucht nur über manche Grenze zu schauen, um sich vorstellen zu können, wie die Entwicklung der Deutschen Dermatologie ohne Otto Braun-Falco und seine Schule hätte verlaufen können. Wenngleich in der Grundlagenforschung oft erfolgreich, spielt die Dermatologie in vielen Ländern der Welt im klinischen Fächerkanon der
Medizin nur noch eine untergeordnete Rolle. Anders bei uns: Das breite klinische Leistungsspektrum der deutschen Dermatologie ist zu großen Teilen auf Initiativen und den unermüdlichen Einsatz sowie die Führungsqualitäten von Otto Braun-Falco zurückzuführen, und nun liegt es an der Schüler- und Enkelgeneration, dieses großartige Erbe zu erhalten und weiter auszubauen.

\section{》) Das breite klinische Leistungsspektrum der deutschen Dermatologie ist zu großen Teilen Otto Braun-Falco zu verdanken}

Der Jubilar wurde am 25.04.1922 in Saarbrücken geboren. Seit 1951 war er mit Sissy Braun-Falco, geborene Golling, verheiratet, die mit ihrer Einfühlungsgabe und souveränen Lebenserfahrung nicht nur ihrem treuen Mann, sondern auch manchem seiner Schüler mit gutem Rat zur Seite stand und die nach ihrem Tod im Februar 2010 unvergessen bleibt. Der Sohn, Prof. Dr. med. Markus Braun-Falco, forscht und lehrt an der früheren Klinik seines Vaters, an der Ludwig-Maximilians-Universität in München.

Die Eckdaten der akademischen Biografie von Otto Braun-Falco sind wohlbekannt - welche Lebensleistung wurde jedoch in dieser Zeit erzielt! Nach den Kriegswirren, die der junge Medizinstudent der Universität Münster/Westfalen - trotz Kriegsdienst und Gefangenschaft glücklicherweise unbeschadet überstand, schloss er das Studium 1948 mit „sehr gut“ an der Universität Mainz ab. Dort erfolg- te auch - „eher zufällig“ -, wie der Jubilar selbst äußerte, der Eintritt in die Mainzer Hautklinik unter Leitung seines Lehrers Prof. Egon Keining, später die Promotion und 1954 die preisgekrönte Habilitation, gefolgt von der Ernennung zum Oberarzt der Hautklinik und 1960 zum außerplanmäßigen Professor.

Bereits 1961 wurde er auf den Lehrstuhl für Dermatologie und Venerologie der Universität Marburg/Lahn berufen und zum Direktor der Universitäts-Hautklinik ernannt. Von 1965 bis 1966 war er Dekan der Marburger Medizinischen Fakultät. Zwischenzeitlich ergangene Rufe auf Lehrstühle an den Universitäten Köln und Heidelberg wurden abgelehnt. Im Jahr 1966 hat Otto Braun-Falco die besonders ehrenvolle und verpflichtende Berufung auf den Lehrstuhl für Dermatologie und Venerologie der Ludwig-Maximilians-Universität in München - in Nachfolge von Prof. Marchionini - angenommen, wo er 1967 seine Tätigkeit als Direktor der großen Klinik zwischen Thalkirchner- und Frauenlobstraße aufnahm. Spätere Rufe an die Universitäten Wien und Zürich, die zu den führenden europäischen Institutionen gehören, wurden von ihm abgelehnt.

Im Jahr 1991 wurde Professor BraunFalco emeritiert (• Abb. 1).

Während seiner aktiven Tätigkeit als Forscher, Lehrer und Kliniker konnte Professor Braun-Falco eine einzigartige Reihe von Erfolgen feiern und hohe Auszeichnungen entgegennehmen. Er erhielt Ehrendoktorwürden der Universitäten Gent, Marburg und Lüttich sowie der Humboldt-Universität zu Berlin. Von den zahl- 


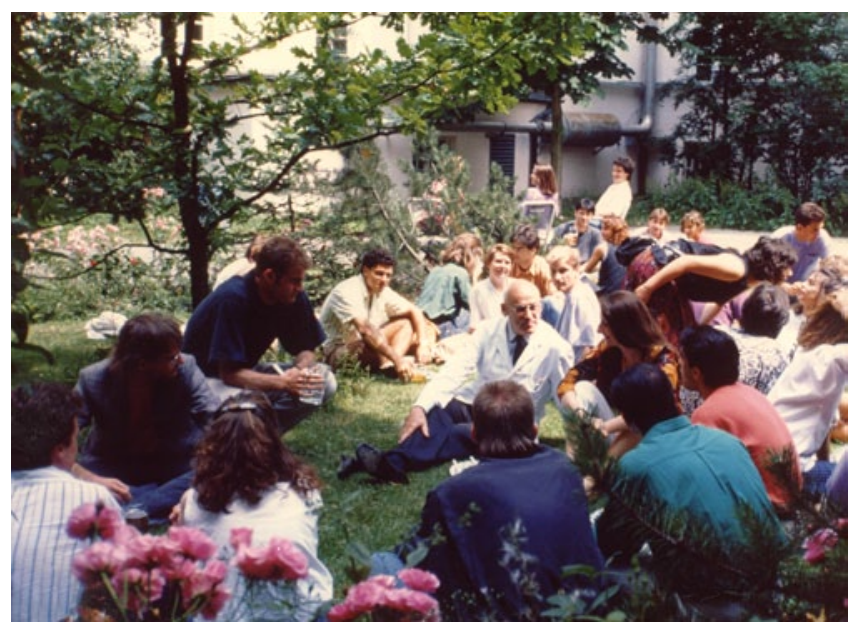

Abb. $1<$ Prof. Otto Braun-Falco im Kreise seiner Münchner Studenten, anlässlich seiner Emeritierung im Jahr 1991. (Mit freundl. Genehmigung Klinik für Dermatologie und Allergologie, LMU München)

reichen Orden, die ihm verliehen wurden, seien der Bayerische Maximiliansorden für Wissenschaft und Kunst, das Große Verdienstkreuz des Verdienstordens der Bundesrepublik Deutschland, der Orden der Aufgehenden Sonne mit Goldenen Strahlen am Halsband auf Empfehlung des Kaisers von Japan, der Bayerische Verdienstorden, die Bayerische Staatsmedaille für Soziale Verdienste sowie die Medaille „München leuchtet“ in Gold genannt.

Für seine wissenschaftliche Tätigkeit erhielt Professor Braun-Falco einige der bedeutendsten Anerkennungen unseres Faches sowie der gesamten Medizin. Nur einige wenige seien hier aufgezählt:

- die Alfred-Marchionini-Medaille in Gold,

- die Herxheimer-Medaille der Deutschen Dermatologischen Gesellschaft,

- die Stephen Rothman-Medaille in Gold der Society for Investigative Dermatology,

- die Hebra-Medaille in Gold der Österreichischen Dermatologischen Gesellschaft,

- die Verdienstmedaille des Berufsverbandes der Deutschen Dermatologen,

- die Cothenius-Medaille in Gold der Deutschen Akademie der Naturforscher Leopoldina zu Halle und

- das Certificate of Appreciation der International League of Dermatological Societies.

Zahlreiche weitere Universitäten und nationale und internationale Gesellschaften verliehen ihm Ehrenmedaillen, und nicht weniger als 37 dermatologische Fachge- sellschaften schmücken sich mit dem Namen Otto Braun-Falco als ihrem Ehrenmitglied.

Die besondere Reputation von Otto Braun-Falco und seine herausragende Stellung im nationalen und internationalen Rahmen wurden durch zahlreiche bedeutende Funktionen reflektiert, die er innehatte. Wiederum seien nur einige hier genannt:

- Von 1977 bis 1982 bekleidete Otto Braun-Falco als Präsident der International League of Dermatological Societies (ILDS) das höchste Amt, das die Weltdermatologie zu vergeben hat,

- von 1982 bis 1985 war er Präsident der Deutschen Dermatologischen Gesellschaft (DDG),

- 1970 Gründungsmitglied der European Society for Dermatological Research (ESDR) und deren Präsident von 1972 bis 1973 ,

- von 1960 bis 1991 Erster Vorsitzender der Münchener Dermatologischen Gesellschaft (MDG) und seither deren Ehrenmitglied,

- 1974 Gründungsmitglied der Arbeitsgemeinschaft Dermatologische Forschung,

- Obmann der Sektion Dermatologie der Akademie der Naturforscher Leopoldina zu Halle, der heutigen Nationalen Akademie der Wissenschaften, und seit 1999 Vorsitzender des AdolfButenandt-Förderkreises für Naturforscher der Leopoldina in München,

- Gründer der Bayerischen AIDS-Stiftung und deren erster Vorsitzender sowie Mitglied im nationalen AIDS-

Beirat der Bundesregierung,

- seit 1988 Mitglied in der Bayerischen

Akademie der Wissenschaften, Mathematisch-Naturwissenschaftliche Klasse und von 1993 bis 1997 deren Sekretär.

Als bedeutendster Repräsentant des Faches Dermatologie, Venerologie und Allergologie war Professor Braun-Falco an wichtigen Kongress- und Fortbildungsaktivitäten in leitender Funktion tätig. So war er in den Jahren 1969 bis 1992 der Leiter der VI.XIII. Fortbildungswochen für praktische Dermatologie und Venerologie der Dermatologischen Klinik der Ludwig-Maximilians-Universität München und begründete ihren Ruf als eine der wichtigsten Fortbildungsveranstaltungen unseres Faches in deutschsprachigen Raum. Er war 1982 Präsident des XVI. World Congress of Dermatology in Tokyo/Japan, 1987 Ehrenpräsident des XVII. World Congress of Dermatology in Berlin, 1988 Präsident der 35. Tagung der Deutschen Dermatologischen Gesellschaft in München und 1997 Ehrenpräsident der 20. Jahrestagung der Vereinigung für Operative und Onkologische Dermatologie, ebenfalls in München.

Die visionäre Weitsicht des Jubilars auf klinischem und wissenschaftlichem Gebiet sowie in der Standespolitik kann nicht genügend hervorgehoben werden und ist retrospektiv durch die Entwicklungen der Dermatologie im Lauf der Jahre eindrucksvoll bestätigt worden: Beispiele sind die Einführung der Lasertechnologie in die Dermatologie, die stetige Förderung der Allergologie und Immundermatologie, der Ausbau der operativen Dermatologie, die Früherkennung der HIV-Problematik und der Notwendigkeit einer integrierten medizinisch sozialen Betreuung betroffener $\mathrm{Pa}$ tienten. Entscheidend für die Förderung des wissenschaftlichen dermatologischen Nachwuchses bis heute waren seine Initiative zur Gründung der Arbeitsgemeinschaft Dermatologische Forschung (ADF) und der ESDR. Besonders hervorzuheben sind auch seine Kontakte auf menschlicher und fachlicher Ebene zu dermatologischen Kollegen und akademischen Institutionen in der damaligen DDR und sein von dem Marchionini-Prinzip „Freundschaft durch Forschung“ geleiteter Brückenschlag zur 


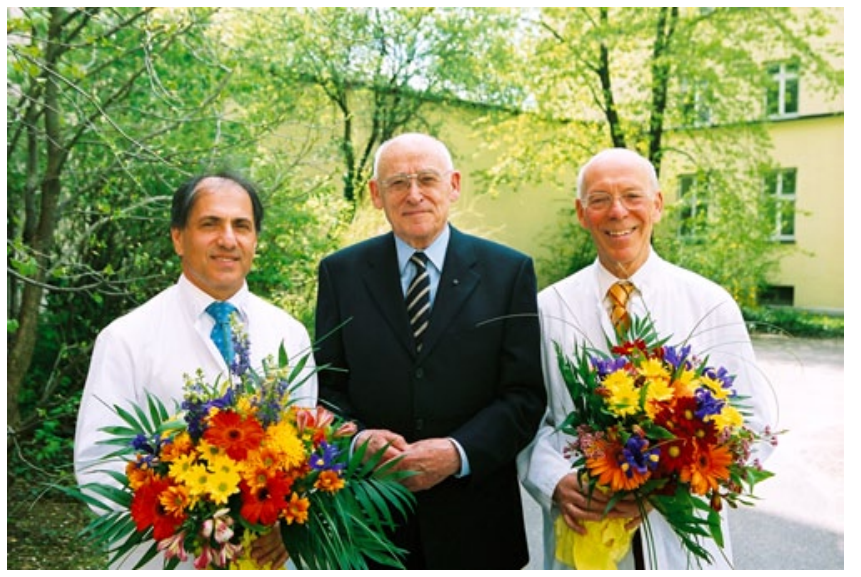

Abb. $2<$ Die Drei von der Derma - Professor Braun-Falco mit seinen Nachfolgern Prof. Dr. med. Dr. h.c. mult. Gerd Plewig sowie Prof. Dr. med. Dr. h.c. Thomas Ruzicka am Tage seiner Inauguration als Nach-Nachfolger von Otto Braun-Falco am 02.05.2006. (Mit freundl. Genehmigung Klinik für Dermatologie und Allergologie, LMU München)

osteuropäischen Dermatologie, insbesondere zu polnischen Kollegen, der zu den an anderer Stelle dieser Festschrift gewürdigten deutsch-polnischen Begegnungen in der Dermatologie und dem „Otto BraunFalco-Stipendium" führte.

Legendär in der gesamten deutschen Medizin ist der mit scherzhafter Bewunderung so genannte „Otto-Versand“: 17 seiner Schüler sind Ordinarien für Dermatologie und Venerologie geworden ( $\bullet$ Abb. 2). Möchte man die Metapher weiterspinnen, so könnte man fast von einem „Ottomatismus" sprechen, mit dem exzellente BraunFalco-Schüler zu Klinikdirektoren geworden sind und den Ruf des Übervaters festigten.

Über die erfolgreiche Schülergeneration wurde bereits bei früheren Anlässen berichtet - nun ist es Zeit, auch der Enkelgeneration zu gedenken, die auch von der Prägung durch Otto Braun-Falco profitieren wie es sich in der deutschen Dermatologie, aber auch international nachweisen lässt: So zählen bedeutende Exponenten der osteuropäischen Dermatologie wie Frau Prof. Sarolta Karpati, Direktorin der Hautklinik der Semmelweis-Universität in Budapest, Prof. Lajos Kemény, Direktor der Hautklinik in Szeged/Ungarn, und Prof. Peter Arenberger, Direktor der Prager Universitätshautklinik und derzeitiger Präsident der Tschechischen Dermatologischen Gesellschaft, ebenso zum erweiterten Kreis der Alumni wie die ehemaligen Otto-BraunFalco-Stipendiaten Prof. Roman Nowitzki in Danzig und PD Dr. Wojciech Baran sowie PD Dr. Adam Reich in Breslau.

Welch reiche Ernte eines 90-jährigen Lebens - eines Lebens in Superlativen! Was sind die persönlichen Geheimnisse, welche sind die Gründe für eine solch einzigartige Laufbahn?

Die Grundvoraussetzungen wie Talent und Intelligenz müssen eine fruchtbare Verbindung mit Enthusiasmus für die Sache, lebenslanger Neugierde und Weitsicht gepaart mit Fleiß und Disziplin eingehen. Diese den visionären Forscher kennzeichnenden Charakteristika werden in der Person von Otto Braun-Falco durch Empathie für seine Patienten, väterliche Fürsorge für seine Mitarbeiter und Begeisterung für seine Aufgabe als Lehrer ergänzt.

Eine unnachahmliche Mischung machte den persönlichen Führungsstil von Otto Braun-Falco aus, den man wegen seiner Einzigartigkeit auch als „ottokratisch“, aber eben nie autokratisch bezeichnen könnte. Er war stets von Autorität und hatte einen Blick für Talente, die er mit einer Mischung aus paternalistischer Fürsorge gepaart mit fachlicher Strenge führte und aufbaute. Der Erfolg gab ihm mit 17 Ordinarien unter seinen Schülern Recht! Die Autorität nahmen ihm seine Mitarbeiter jederzeit ab - basierte sie doch nicht auf Amt und Chefposition, sondern auf überragendem Wissen und Führungspersönlichkeit. Exzellenten Mitarbeitern gegenüber entwickelte er eine Toleranz und ein Laisser-faire, welche eine freie Entwicklung zuließen. So konnte sich in der Münchner Dermatologischen Klinik eine inspirierende Atmosphäre aus Teamgeist und Individualismus ausbilden, die den fruchtbaren Boden für den Erfolg der Braun-Falco-Klinik bereitete.

Otto Braun-Falco ist jedoch nicht nur ein „homo dermatologicus“; zum Faszinosum seiner Persönlichkeit gehören auch Esprit und Charme, charismatische Aura, soigniertes Äußeres und bescheidenes, wenn auch immer souveränes Auftreten. Sie runden das Bild eines Grandseigneurs und einer außergewöhnlichen Galionsfigur ab. Ein glückliches Familienleben, eine loyale liebende Ehefrau, ein talentierter Sohn, der den dermatologischen Stafettenstab übernahm, und der Optimismus, mit dem Otto Braun-Falco den herben Schicksalsschlägen trotzte, bildeten die Grundlage, auf der sich der berufliche Erfolg und seine Lebensleistung entfalten konnten.

Anlässlich von naturgemäß etwas seltener werdenden persönlichen Treffen, aber häufigen telefonischen Kontakten mit dem Jubilar können die beiden Autoren ebenso wie viele seiner Schüler und Weggefährten unverändert die einmalige OBF-Mischung aus beruflicher Exzellenz und Lebensweisheit - gepaart mit Humor und persönlicher Zuwendung - erleben, die uns dankbar macht für das Privileg und Glück, einen akademischen Lehrer und Mentor wie Otto Braun-Falco zu haben.

Lieber Otto, Deine weltweite Fangemeinde - Deine „Ottomanen“ - gratuliert Dir aufs Herzlichste zu Deinem 90. Geburtstag und bringt Dir mit diesem Sonderheft von Der Hautarzt ein persönliches Geschenk, das wir im Namen des Herausgeberteams überreichen. Wir wünschen Dir von Herzen alles Gute und viele weitere glückliche Jahre im Kreise Deiner dankbaren und auf Dich stolzen Dermatologenfamilie!

Prof. Dr. med. Dr. h.c. Thomas Ruzicka

Prof. Dr. med. Michael Meurer

\section{Korrespondenzadressen}

Prof. Dr. Dr. h.c. T. Ruzicka

Klinik und Poliklinik für Dermatologie und Allergologie, Klinikum der Ludwig-MaximiliansUniversität München

Frauenlobstr. 9-11, 80337 München

Thomas.Ruzicka@med.uni-muenchen.de

\section{Prof. Dr. M. Meurer}

Klinik und Poliklinik für Dermatologie, Universitäts Allergie Centrum, Technische Universität Dresden

Fetscherstr. 74, 01307 Dresden michael.meurer@uniklinikum-dresden.de 